\title{
The Effect of Ethanol on Hydroxyl and Carbonyl Groups in Biopolyol Produced by Hydrothermal Liquefaction of Loblolly Pine: ${ }^{31}$ P-NMR and ${ }^{19}$ F-NMR Analysis
}

Yusuf Celikbag ${ }^{1}$, Brian K. Via ${ }^{1,2,3^{*}}$, Sushil Adhikari ${ }^{2,3}$, Gisela Buschle-Diller ${ }^{3}$, Maria L. Auad ${ }^{4}$

${ }^{1}$ School of Forestry and Wildlife Sciences, Auburn University, Auburn, AL. 36849 USA

${ }^{2}$ Center for Bioenergy and Bioproducts, Auburn University, Auburn, AL 36849 USA

${ }^{3}$ Department of Biosystem Engineering. Auburn University, Auburn, AL. 36849 USA

${ }^{4}$ Department of Chemical Engineering. Auburn University, Auburn, AL. 36849 USA

* Corresponding author at: Auburn University, Forest Products Development Center, School of Forestry and Wildlife Sciences, Auburn, AL 36849, USA. Tel.: +1-334-844-1088; Fax: +1-334844-1088. E-mail address: bkv0003@auburn.edu (B.K.Via).

Page 1 of 30

(C) 2016. This manuscript version is made available under the Elsevier user license http://www.elsevier.com/open-access/userlicense/1.0/ 


\begin{abstract}
The goal of this study was to investigate the role of ethanol and temperature on the hydroxyl and carbonyl groups in biopolyol produced from hydrothermal liquefaction of loblolly pine (Pinus spp) carried out at 250, 300, 350 and $390^{\circ} \mathrm{C}$ for $30 \mathrm{~min}$. Water and water/ethanol mixture $(1 / 1, w t / w t)$ were used as liquefying solvent in the HTL experiments. HTL in water and water/ethanol is donated as W-HTL and W/E-HTL, respectively. It was found that $300{ }^{\circ} \mathrm{C}$ and water/ethanol solvent was the optimum liquefaction temperature and solvent, yielding up to 68.1 wt.\% bio-oil and 2.4 wt.\% solid residue. ${ }^{31} \mathrm{P}-\mathrm{NMR}$ analysis showed that biopolyol produced by W-HTL was rich in phenolic OH while W/E- HTL produced more aliphatic OH rich biopolyols. Moreover, biopolyols with higher hydroxyl concentration were produced by W/E-HTL.

Carbonyl groups were analyzed by ${ }^{19} \mathrm{~F}-\mathrm{NMR}$, which showed that ethanol reduced the concentration of carbonyl groups.
\end{abstract}

Keywords: Biopolyol, Bio-oil, Hydrothermal liquefaction, ${ }^{31} \mathrm{P}-\mathrm{NMR},{ }^{19} \mathrm{~F}-\mathrm{NMR}$. 


\section{Introduction}

Lignocellulosic biomass is being used as an alternative feedstock for the production of bio-based polyols (biopolyols) in order to reduce our dependency on petroleum. Polyol is a compound that has multiple hydroxyl groups in its structure and is essential for the synthesis of a variety of polymers such as epoxy, polyurethane, polyester, polycarbonate, and phenolic resin. However, today the major source for polyols is petroleum. Researchers have thus focused on biopolyol in the face of depleting petroleum reserves, increased greenhouse effect, as well as an increasing demand by the consumers for bio-based products. Moreover, the U.S. Department of Energy (DOE) and the U.S. Department of Agriculture (USDA) have prioritized the development of bioenergy and bioproducts with the goal to produce $18 \%$ of the current U.S. chemical commodities from lignocellulosic biomass by 2020, and 25\% by 2030 (Perlack et al. 2005).

Bio-oil, the black liquid produced by degradation of lignocellulosic biomass through thermomechanical processes, has attracted interest to be used as a biopolyol because of its high hydroxyl concentration. Gas chromatography-mass spectrometry (GC-MS) analysis of bio-oil showed that bio-oil is a highly complex mixture, mainly composed of carbohydrates, furans, phenols, guaiacols, syringols type of compounds (Thangalazhy-Gopakumar et al. 2010). Hydrothermal liquefaction (HTL), also known as direct liquefaction, is a thermomechanical conversion technique to produce bio-oil where lignocellulosic biomass is converted into liquid (bio-oil), gas and solid products using subcritical or supercritical water at elevated temperature $\left(250-370{ }^{\circ} \mathrm{C}\right)$ and pressure $(2-24 \mathrm{MPa})$. Bio-oil produced by the HTL process has a significant potential for commercialization in terms of price and life cycle assessment (Elliott et al. 2015). The effect of process parameters such as temperature, pressure, reaction time and feedstock type 
on the bio-oil yield in HTL process has been intensively studied and valuable literature reviews can be found elsewhere (Akhtar and Amin 2011, Toor et al. 2011). Approximately $30-40$ wt.\% bio-oil (based on dry mass of biomass) is obtained from the hydrothermal liquefaction of lignocellulosic biomass (Akhtar and Amin 2011). Recently, ethanol has received attention to be used as a co-solvent in the HTL process due to its high hydrogen solvent capability. Yuan et al. (2007) proposed that the highly reactive free radicals generated from the HTL of biomass were stabilized by the ethanol which acts as a hydrogen-donor solvent, and Cheng et al. (2010) reported that bio-oil yield could be increased from 40 to $65 \mathrm{wt} . \%$ when ethanol was used along with water in a sub-critical condition. In another study, alkaline lignin was liquefied in hot compressed water/ethanol medium, and found that addition of ethanol increased the degradation of lignin which resulted in a lower amount of solid residue (Yuan et al. 2010). This improvement may be due to the low dielectric constant of ethanol which facilitates dissolving of high molecular weight lignin at supercritical temperatures (Krammer and Vogel 2000). The synergistic effect of water/ethanol mixture in the liquefaction of rice husk for bio-oil production via the HTL process was also observed by Liu et al. (2013). Besides the high bio-oil yield, the addition of ethanol to water also affects the distribution of phenolics such as phenol, ethylphenol and guaiacols, ethylguaiacol and syringol in the bio-oil as well (Ye et al. 2012, Ouyang et al. 2015). Recently, Kosinkova et al. (2015) reported that aqueous ethanol improved the higher heating value (HHV) of bio-oil to be used in the field of biodiesel applications. Moreover, the addition of medical stone as a catalyst into aqueous ethanol could further increase the yield of bio-oil produced by HTL of cotton seed (Yan et al. 2015).

Compounds containing hydroxyl and carbonyl groups in bio-oil play a major role in its utilization as a biopolyol. There are a variety of polymers synthesized using bio-oil including 
phenolic resin (Choi et al. 2015), polyurethane (Hu and Li 2014), epoxy (Kuo et al. 2014) and polyester (Yu et al. 2006), and the hydroxyl groups in bio-oil are utilized to synthesize these polymers. Wei et al. (2014) used bio-oil to modify an epoxy resin, and found that hydroxyl number (OHN) of the bio-oil had a significant effect on the glass transition temperature (Tg) of the modified epoxy resin. In another study, bio-oil was used to synthesize a new adhesive system for application in particle boards. Here, the hydroxyl groups in bio-oil contributed to the condensation reactions with melamine-formaldehyde and melamine-urea-formaldehyde resin precursors which ultimately affected the properties of the resulting resin system (Kunaver et al. 2010). Zou et al. (2012) synthesized a polyurethane foam using bio-oil, and reported that a high number of primary hydroxyl groups from the bio-oil was necessary to increase the mechanical and thermal properties of polyurethane foam. Thus, it could be hypothesized that the reaction behavior and the mechanical/thermal properties of the resulting polymer depend on the interaction between hydroxyl groups in bio-oil and the formaldehyde for phenol formaldehyde resin, isocyanate for the polyurethane, epoxide groups for epoxy resin, and ester groups for polyester production.

Compounds containing carbonyl groups in the bio-oil such as aldehydes, ketones and quinones are found to play a major role in the aging of bio-oil during storage (Czernik et al. 1994). Reactions between compounds containing carbonyl and hydroxyl groups in the bio-oil have been reported to be a reason for bio-oil aging which results in the polymerization and consequently a change in viscosity (Czernik et al. 1994). The production of carboxylic acid as a result of oxidation of carbonyl groups is another problem which may cause equipment corrosion. Most recently, our group has studied the curing reaction of a commercial epoxy resin (EPON828) with a bio-oil, and found that carbonyl groups in the bio-oil contributed the curing of 
epoxy resin along with the hydroxyl groups as well (Celikbag et al. 2015). Therefore, the characterization and quantification of hydroxyl and carbonyl groups in the bio-oil is crucial to determine its quality as well as optimization for bio-oil based polymer synthesis.

The literature on liquefaction of lignocellulosic biomass in water/ethanol mixture via HTL process lacks specific information regarding the source and variation of hydroxyl and carbonyl groups in the bio-oil. Characterization of these functional groups plays an important role in the improvement of bio-oil for its effective utilization as a biopolyol. Therefore, a comprehensive hydroxyl and carbonyl group analysis is necessary to understand the source and variation of bio-oil hydroxyl and carbonyl group content which will make it possible to engineer the properties of bio-based polymers and their synthesis in future studies. To the best of our knowledge, this is the first study that has used the ${ }^{31} \mathrm{P}-\mathrm{NMR}$ and ${ }^{19} \mathrm{~F}-\mathrm{NMR}$ analytical tools to partition out the hydroxyl and carbonyl groups to understand the role of ethanol in the HTL process of lignocellulosic biomass. Thus, the objectives of this study were to (i) investigate the effect of ethanol and liquefaction temperature and on hydroxyl and carbonyl groups, and (ii) analyze the variation and source of these functional groups in the bio-oil using ${ }^{31} \mathrm{P}-\mathrm{NMR}$ and ${ }^{19} \mathrm{~F}-$ NMR.

\section{Materials and Methods}

\subsection{Materials}

Loblolly pine wood chips that are free from bark and leaf were obtained from a local chipping plant in Opelika, AL, USA. Loblolly pine composition (48.5\% cellulose, 31.3\% hemicellulose, $25.8 \%$ lignin and $4.5 \%$ extractives) was determined using standard wet chemistry analysis protocol. All chemicals were purchased from VWR; except the phosphorylating agent 
for ${ }^{31} \mathrm{P}-\mathrm{NMR}$ analysis, 2-chloro-4,4,5,5-tetramethyl-1,3,2-dioxaphospholane (TMDP), purchased from Sigma Aldrich. All chemicals were reagent grade and used as received.

\subsection{Hydrothermal Liquefaction (HTL) of Loblolly Pine}

Loblolly pine was ground by a hammer mill (New Holland grinder model 358, New Holland, PA.) using 40 mesh sieve size for particle size reduction, and the pine passed through 40 mesh were used. HTL was carried out in $1 \mathrm{~L}$ high pressure/high temperature Parr® Reactor (Parr Instrument Company, Model 4577 HP/HT pressure reactor, Moline, IL., USA) equipped with mechanical stirrer and reactor controller (Parr Instrument Company, Model 4848, Moline, IL., USA). The reactor was charged with $25 \mathrm{~g}$ of 40 mesh loblolly pine and $250 \mathrm{~g}$ of solvent (biomass/solvent=1/10, wt/wt) which consisted of either DI water or DI water/ethanol mixture $(1 / 1, \mathrm{wt} / \mathrm{wt})$. The HTL process with water and water/ethanol is denoted as W-HTL and W/EHTL, respectively. Before heating up the reactor, high purity nitrogen was purged into the reactor to remove the air, and then reactor was pressurized to $2 \mathrm{MPa}$ with nitrogen to force all the reactive materials into the liquid phase. Hydrothermal liquefaction was carried out at $300 \mathrm{rpm}$ for 30 min. at four different conditions: $250^{\circ} \mathrm{C}$ (far-critical), $300{ }^{\circ} \mathrm{C}$ (sub-critical), $350{ }^{\circ} \mathrm{C}$ (nearcritical), and $390^{\circ} \mathrm{C}$ (super-critical). HTL time was started once the set temperature was reached. For the $390^{\circ} \mathrm{C}$ super-critical condition, the reactor was not initially pressurized to $2 \mathrm{MPa}$ with nitrogen due to the safety limit of the reactor. The final pressure in the reactor at each temperature was read from the reactor controller and is shown in Table 1. A typical temperaturepressure profile during HTL experiment is illustrated in Fig. S1 (see supporting information). After 30 min. reaction, the reactor is immersed in iced water to quench the reaction.

\subsection{Product Separation and Calculation of the Product Yields}


The procedure of product separation is illustrated in Fig. 1. Once the reactor was cooled down to $24^{\circ} \mathrm{C}$, the gas fraction was released, and the reactor was opened. The composition of the gas fraction was not analyzed in this study. The amount of gas produced was calculated by the weight difference of the reactor after venting the gas (Eq. 1), where W1 is the weight of the reactor before HTL containing biomass and solvent; and W2 is the weight of the reactor (containing liquefied biomass) after HTL and venting the gas. The slurry (liquefied wood) was poured into a flask, and the reactor was rinsed with dichloromethane (DCM) to collect all the contents in the reactor. The slurry was then diluted with $400 \mathrm{~mL}$ DCM. The solid and the liquid part in the slurry was vacuum filtered using preweighed Whatmann\#5 filter paper. The solid part, accumulated on the filter paper, was oven dried at $105^{\circ} \mathrm{C}$ for overnight, and assigned as "residue". The yield of residue was calculated using Eq. 2. The liquid part was poured into a $1 \mathrm{~L}$ separatory funnel to isolate the organic and the aqueous phases. Since water is not miscible in DCM, organic and aqueous products separated naturally in the separatory funnel. The organic products were carefully transferred to a rotary evaporator. The DCM and the solvent (water and/or ethanol) retained in the organic phase was removed by rotary evaporation at $65{ }^{\circ} \mathrm{C}$. The DCM soluble compounds, a viscous-black liquid, were collected in the flask, which was donated as "bio-oil", and the yield of bio-oil was calculated using Eq. 3. The aqueous products (water and water soluble products) accumulated in the top layer, and assigned as "light oil". Since the major interest of this study was the bio-oil, the light oil was not further analyzed in this study. Quantification of light-oil is one challenge in HTL studies due to the high boiling point of water (Cheng et al. 2010). Therefore, the yield of the light oil was calculated by difference using Eq. 4 for simplicity. However, it should be noted that calculating the yield of light-oil by difference results experimental errors. All experiments were carried out in duplicates and ANOVA was 
performed at $95 \%$ confidence interval to find out whether the results were statistically significant.

Gas yield $($ wt. $\%)=\frac{W 1-W 2}{\text { wt.of the loblolly pine }(\text { dry basis })} \times 100$

Residue yield $(w t . \%)=\frac{w t . o f \text { the solid products }}{\text { wt.of the loblolly pine }(\text { dry basis })} \times 100$

Bio - oil yield $(w t . \%)=\frac{\text { wt.of the bio-oil after rotary evaporator }}{\text { wt.of the loblolly pine }(\text { dry basis })} \times 100$

Light oil yield $(w t . \%)=100-$ Yield $_{\text {Gas }}$ residue+bio-oil

\subsection{Hydroxyl (OH) Group Analysis of Bio-oil: ${ }^{31} \mathrm{P}-\mathrm{NMR}$}

${ }^{31} \mathrm{P}-\mathrm{NMR}$ provides great deal of information to determine the content of $\mathrm{OH}$ groups present in the bio-oil. In this technique, $\mathrm{OH}$ groups belonging to aliphatic, phenolic, carboxylic units were phosphitylated by TMDP followed by quantitative ${ }^{31} \mathrm{P}-\mathrm{NMR}$. In the phosphitylation reaction, TMDP reacted with the free $\mathrm{OH}$ groups in the bio-oil, and yielded the derivatized compound and hydrochloric acid $(\mathrm{HCl})$. Phosphitylation of bio-oil was performed according to Celikbag et al. (2015), and ${ }^{31} \mathrm{P}-\mathrm{NMR}$ spectra were acquired with a Bruker Avance II $250 \mathrm{MHz}$ spectrometer using inverse gated decoupling pulse sequence, $90^{\circ}$ pulse angle, $25 \mathrm{~s}$ pulse delay and 128 scans following the methods of Ben and Ragauskas (2011). Detailed method is presented in the Supporting Information. All experiments were performed in duplicates with ANOVA at $95 \%$ confidence interval.

\subsection{Carbonyl Groups Analysis of Bio-oil: ${ }^{19}$ F-NMR}

The carbonyl groups in bio-oil was quantitatively determined by ${ }^{19} \mathrm{~F}-\mathrm{NMR}$ according to the method reported by Huang et al. (2014). In this technique, the carbonyl groups (aldehydes, 
ketones and quinones) in the bio-oil were derivatized with 4-(tri-fluoromethyl)phenylhydrazine. For quantitative analysis, 3-trifluoromethoxybenzoic was used as the internal standard. Detailed method is presented in the Supporting Information. ${ }^{19}$ F-NMR spectra were acquired with a Bruker Avance II $250 \mathrm{MHz}$ spectrometer using $90^{\circ}$ pulse angle without proton decoupling, $10 \mathrm{~s}$ pulse delay and 400 scans. All experiments were performed in triplicates and ANOVA was performed at $95 \%$ confidence interval.

\section{Results and Discussion}

\subsection{Product Yields}

\subsubsection{Effect of Temperature on Product Yields}

Product yields obtained under different HTL conditions are illustrated in Fig. 2. It was found that temperature had a significant effect on bio-oil yield ( $p$-value $=0.0007$ and $<0.0001$ for W-HTL and W/E-HTL, respectively), and residue yield ( $p$-value $=0.0001$ and $<0.0001$ for WHTL and W/E-HTL, respectively). The maximum bio-oil yield of $68 \mathrm{wt} . \%$ and minimum residue yield of 2 wt. $\%$ was obtained at W/E-HTL at $300{ }^{\circ} \mathrm{C}$. The bio-oil yield first increased when the temperature increased from $250{ }^{\circ} \mathrm{C}$ to $300^{\circ} \mathrm{C}$, and then decreased as the temperature further increased to $390{ }^{\circ} \mathrm{C}$. The first increase in the bio-oil yield could be attributable to enhanced degradation of lignocellulosic biomass into bio-oil due to the elevated temperature. The minimum temperature for hemicellulose decomposition in water was reported to be $180{ }^{\circ} \mathrm{C}$ (Changi et al. 2015), and higher temperatures $\left(>260^{\circ} \mathrm{C}\right.$ ) are required to overcome the activation energy for lignin degradation (Yuan et al. 2010); therefore, it is reasonable that $\mathrm{HTL}$ at $300{ }^{\circ} \mathrm{C}$ resulted in higher degradation of biomass than at $250^{\circ} \mathrm{C}$, and consequently led to increased biooil yield. However, bio-oil yield decreased while residue and gas yield increased as the HTL 
temperature increased to $390^{\circ} \mathrm{C}$ as can be seen in Fig. 2. Many of the HTL studies reported similar trends regarding a critical temperature for HTL of lignocellulosic biomass at which biooil yield begins to drop and more solid residue and gaseous products are produced at above the critical temperature (Akhtar and Amin 2011). In this study, $300{ }^{\circ} \mathrm{C}$ was found to be the best temperature in terms of bio-oil and residue yield for both W-HTL and W/E-HTL. A kinetic study also showed that gaseous products and solid residue are formed by the secondary reactions of bio-oil at higher temperatures as a result of cracking and recondensation of bio-oil (Valdez and Savage 2013). Thus, it is possible that the decrease in bio-oil yield at higher temperatures is attributable to the enhanced cracking and condensation reactions. The decrease in the dielectric constant $(\varepsilon)$ of water at higher temperature and pressure could be another reason for the decrease in bio-oil yield. Since the dielectric constant of water measures the polarity, it could be defined as the rough measurement of water's ability to dissolve polar compounds, and it decreases from 27.1 at subcritical conditions $\left(150^{\circ} \mathrm{C}<\mathrm{T}<350^{\circ} \mathrm{C}, 0.4 \mathrm{MPa}<\mathrm{p}<20 \mathrm{MPa}\right)$ to 5.9 at supercritical condition $\left(\mathrm{T}>370^{\circ} \mathrm{C}, \mathrm{p}=25 \mathrm{MPa}\right)(\mathrm{Krammer}$ and Vogel 2000). Moreover, water at subcritical conditions acts like an acid/base catalyst precursor which is desirable for biomass liquefaction while water at supercritical conditions exhibits more like a non-polar solvent properties (Kruse and Dinjus 2007). Therefore, it could also be conjectured that the lower dielectric constant of water at higher temperatures could have contributed to lower bio-oil yield observed in this study.

\subsubsection{Effect of Ethanol on Product Yields}

ANOVA analysis with 95\% confidence interval showed that addition of ethanol significantly increased the bio-oil yield obtained at each HTL temperature ( $p$-value $=0.0016$, $0.0017,0.0076$ and 0.0014 for $250,300,350$ and $390{ }^{\circ} \mathrm{C}$, respectively), and decreased the amount of residue generated during the HTL ( $p$-value $=0.0063,<0.0001,0.0027$ and 0.0003 for 
250, 300, 350 and $390{ }^{\circ} \mathrm{C}$, respectively). All the W/E-HTL experiments exhibited higher bio-oil yield and lower residue yield than the W-HTL experiments; in fact, the lowest bio-oil yield (30 \pm 0.5 wt.\%) obtained from W/E- HTL was even higher than the maximum bio-oil yield (26 \pm 1.6 wt.\%) obtained from the W-HTL suggesting that water/ethanol shows great synergy effect for HTL of the lignocellulosic biomass. Moreover, much lower solid residue (2 wt.\%) was produced by W/E- $\mathrm{HTL}$ at $300{ }^{\circ} \mathrm{C}$ while $\mathrm{W}-\mathrm{HTL}$ produced $39 \mathrm{wt} . \%$ solid residue at $300^{\circ} \mathrm{C}$ which indicates that addition of ethanol effectively inhibited the re-condensation of degradation products of lignocellulosic biomass. During the HTL, biomass is degraded to fragments and free radicals are generated. When there is no hydrogen donor solvent, these reactive free radicals recombines and forms high molecular weight products called solid residue or char. In case a sufficiently high amount of a hydrogen donor solvent such as ethanol is introduced to the HTL, free radicals can be stabilized and the formation of solid residue is decreased (Vasilakos and Austgen 1985). Thus, lower residue content at W/E-HTL is attributable to the hydrogen donor capability of ethanol. As Liu et al. (2013) summarized the synergetic effect of water/ethanol, the high bio-oil and low residue yield from W/E-HTL experiments could be attributed to (i) enhanced hydrogen donor capability of ethanol at subcritical and supercritical conditions and acting as a reaction substrate, (ii) high ability of ethanol to dissolve oily products, (iii) ability of ethanol to stabilize the free radicals resulting in lower residue content, and (iv) the increased solubility of high molecular weight products in water/ethanol mixture at subcritical conditions. In addition to the synergistic effects mentioned above, conversion of highly reactive carbonyl groups in the bio-oil to more stable acetal groups by ethanol could be another reason for the low residue content in W/E- HTL. Compounds containing carbonyl groups such as aldehydes and ketones are primarily responsible for the repolymerization of bio-oil due to their high reactivity and consequently 
result in the generation of solid residue as well as an increase in the viscosity of bio-oil (Czernik et al. 1994). In this study, it was found that bio-oil produced from W/E- HTL had a lower carbonyl concentration (see below, section 3.3. ${ }^{19} \mathrm{~F}-\mathrm{NMR}$ analysis of bio-oil) suggesting that ethanol stabilized the carbonyl groups, and as a result, a lower amount of solid residue was generated.

\subsection{Hydroxyl (OH) Group Analysis of Bio-oil: ${ }^{31} \mathrm{P}-\mathrm{NMR}$}

The OHNs of all bio-oils produced at different HTL conditions are illustrated in Fig. 3. A typical ${ }^{31} \mathrm{P}-\mathrm{NMR}$ spectrum of a bio-oil is shown in Fig. S2 (see supporting information). The type of $\mathrm{OH}$ groups including aliphatic, phenolic (guaiacyl, catechol, $\mathrm{C}_{5}$ substituted condensed phenolic types) and acidic type of $\mathrm{OH}$ were identified according to ${ }^{31} \mathrm{P}-\mathrm{NMR}$ chemical shifts reported by Ben and Ragauskas (2011). High OHN (5.35-10.70 mmol/g) is required for a bio-oil to be considered as a high quality biopolyol (Zou et al. 2012). All the bio-oils, except the bio-oils produced from $\mathrm{W}-\mathrm{HTL}$ at $300^{\circ} \mathrm{C}$ and $350^{\circ} \mathrm{C}$, exhibited higher $\mathrm{OHN}$ than $5.35 \mathrm{mmol} / \mathrm{g}$; therefore, could thus be considered as a good candidates as biopolyols.

\subsubsection{Effect of Temperature on Hydroxyl Groups}

ANOVA analysis showed that temperature had a significant effect on the total OHN of bio-oils produced by both W-HTL ( $p$-value $=0.0007$ ) and W/E-HTL ( $p$-value $=0.0015)$. In the case of W-HTL, the total OHN first decreased $(p$-value $=0.0142$ ) as the temperature was increased from 250 to $300{ }^{\circ} \mathrm{C}$, and then increased $\left(p\right.$-value $=0.0873$ and 0.0088 for $300-350{ }^{\circ} \mathrm{C}$ and $350-390{ }^{\circ} \mathrm{C}$, respectively) with the increasing temperature to $390^{\circ} \mathrm{C}$. On the other hand, the total OHN of bio-oils from W/E-HTL experiments decreased steadily ( $p$-value $=0.0782$ and 0.0285 for $250-300{ }^{\circ} \mathrm{C}$ and $300-350^{\circ} \mathrm{C}$, respectively) till $350^{\circ} \mathrm{C}$, and then started to increase ( $p$ - 
value $=0.3759$ ) as the temperature reached to $390{ }^{\circ} \mathrm{C}$. A similar trend in the $\mathrm{OHN}$ with temperature was observed for the bio-oils produced by organic solvent liquefaction of biomass where authors attributed this trend to condensation reactions that occurred between degradation fragments of biomass (Wei et al. 2014). It is noteworthy to mention that for the both W-HTL and W/E-HTL experiments, aliphatic $\mathrm{OH}$ decreased while phenolic $\mathrm{OH}$ increased with increasing HTL temperature from 250 to $390{ }^{\circ} \mathrm{C}$. Aliphatic $\mathrm{OH}$ was the dominant hydroxyl type at the lower temperature; however, at higher temperature, phenolic type $\mathrm{OH}$ was observed to be the major hydroxyl type. For instance, at $250{ }^{\circ} \mathrm{C} \mathrm{HTL}$, the aliphatic $\mathrm{OH}$ accounted for $50.3 \%$ and $62.7 \%$ of the total OHN of W-HTL and W/E-HTL, respectively. On the other hand, when the HTL temperature reached to $390{ }^{\circ} \mathrm{C}$, phenolic $\mathrm{OH}$ accounted for $91 \%$ and $75.6 \%$ of the total OHN of W-HTL and W/E-HTL, respectively. Statistical analysis also showed that HTL temperature had significant effect on aliphatic and phenolic $\mathrm{OH}$ ( $\mathrm{p}$-value $<0.0001$ for both aliphatic and phenolic OH at W-HTL and W/E-HTL). This trend (decrease in the aliphatic OH and increase in the phenolic $\mathrm{OH}$ with increasing HTL temperature) could be due to the thermal stabilities of wood polymers (cellulose, hemicellulose and lignin). The higher concentration of aliphatic $\mathrm{OH}$ at lower temperature may be attributable to mainly hemicellulose degradation since (i) hemicellulose is the first depolymerized wood polymer under heat (Via et al. 2013), and (ii) the degradation route of hemicellulose in water yields sugar derivatives containing aliphatic $\mathrm{OH}$ groups (Changi et al. 2015). Lignin is the main source of the phenolic compounds in a bio-oil (Cheng et al. 2010), and degradation of lignin occurs at temperature above $260^{\circ} \mathrm{C}$ (Yuan et al. 2010). Thus, the increase in the amount of phenolic $\mathrm{OH}$ with increasing HTL temperature could mostly be due to the lignin degradation. The acidic $\mathrm{OH}$ did not significantly changed with temperature ( $\mathrm{p}$-value $=0.2980$ and 0.0462 for W-HTL and W/E-HTL, respectively), and stayed at 
a concentration of $0.66 \pm 0.06$ and $0.72 \pm 0.16 \mathrm{mmol} / \mathrm{g}$ for W-HTL and W/E-HTL, respectively.

Changi et al. (2015) reviewed the degradation of cellulose and hemicellulose in water at sub- and super-critical conditions. Authors reported that at sub-critical conditions, hemicellulose degradation occurs much faster than cellulose while cellulose degradation increases when the HTL conditions are at the near- or super-critical conditions. It was also reported that their degradation products contain acidic components such as formic, acetic and lactic acid. Therefore, degradation products of hemicellulose and cellulose could be attributable to the source of acidic $\mathrm{OH}$ at subcritical $\left(250-350^{\circ} \mathrm{C}\right)$ and super-critical $\left(390{ }^{\circ} \mathrm{C}\right)$ conditions, respectively.

\subsubsection{Effect of Ethanol on Hydroxyl Groups}

All of the bio-oils obtained from W/E-HTL experiments at each temperature exhibited higher total OHN than W-HTL except at $390^{\circ} \mathrm{C}$. Ethanol was found to have a significant effect on the total $\mathrm{OHN}$ of the bio-oils produced at 250,300 and $350{ }^{\circ} \mathrm{C}(p$-value $=0.0123,0.0052$ and 0.0193, respectively); however, no significant effect was observed for the experiment at $390^{\circ} \mathrm{C}$ ( $p$-value $=0.1082)$. The high OHN at W/E-HTL experiments could be attributed to the synergy effect of water and ethanol as discussed in the section 3.1.2. Since ethanol is a good hydrogen donor solvent and effectively inhibits the re-condensation of biomass degradation products, the bio-oil obtained from W/E-HTL had a lower molecular weight and a higher polydispersity index (Cheng et al. 2010) suggesting that the bio-oil mainly contains monomers and oligomers from decomposition of biomass. Therefore it is conjectured that the higher amount of monomers and oligomers provided more side groups such as hydroxyls in the bio-oil. 
One major result found in this study was that the bio-oils produced by W/E-HTL at each temperature contain more aliphatic and less phenolic $\mathrm{OH}$ than the bio-oils obtained from $\mathrm{W}$ HTL. This finding suggests that ethanol significantly contributed the aliphatic type $\mathrm{OH}$ in the bio-oils (p-value $=0.0050,0.0003,0.0005$ and 0.0107 for $250,300,350$ and $390^{\circ} \mathrm{C}$, respectively). Our lab has previously studied the source and variation of hydroxyl groups of the bio-oil produced by liquefaction of loblolly pine with ethylene glycol (EG), and found that lignin fragments undergo condensation reaction with EG which results in less phenolic and more aliphatic type of hydroxyl groups (Celikbag et al. 2014). The same rational can be applied to the W/E-HTL in which ethanol may undergo condensation reactions with the phenolic $\mathrm{OH}$ groups of the lignin fragments. This finding is also in agreement with previous GC/MS studies of cornstalk lignin in W/E-HTL where the yields of phenolic compounds (phenol, ethylphenol, guaiacol) were reported to be lower when the ethanol concentration in the water/ethanol mixture was increased (Ye et al. 2012). ANOVA analysis of phenolic OH showed that ethanol had significant effect at $390{ }^{\circ} \mathrm{C}$ only ( $p$-value $=0.0033$ ). For below supercritical conditions, no significant effect of ethanol was observed on phenolic $\mathrm{OH}$ ( $p$-value $=0.5963,0.1555$ and 0.2712 for 250,300 and $350^{\circ} \mathrm{C}$, respectively).

These findings suggest that OHN of a bio-oil could be adjusted by HTL temperature and ethanol. For example, W/E-HTL at lower temperature seems to constitute optimum HTL conditions for the production of biopolyol with high concentration of aliphatic OH. Such biopolyols could be utilized in polyester production. On the other hand, bio-oil produced from high temperature W-HTL offers high concentration of phenolic $\mathrm{OH}$ which could be used as a biopolyol for the production of rigid polymers such as epoxy. However, the bio-oil yield should 
be taken into account as well because it is an important consideration for the economic viability of the process.

\subsection{Carbonyl (C=O) Group Analysis of Bio-oil: ${ }^{19}$ F-NMR}

\subsubsection{Effect of Temperature on Carbonyl $(C=0)$ Groups}

The carbonyl group concentration of bio-oil derivatized with 4-(tri-

fluoromethyl)phenylhydrazine was calculated by quantitative ${ }^{19} \mathrm{~F}-\mathrm{NMR}$ and results are illustrated in Fig. 4. A typical ${ }^{19}$ F-NMR spectra is shown in Fig. S3 (see supporting information). ANOVA analysis showed that temperature had no statistically significant effect on the carbonyl concentration in the bio-oil produced from W-HTL ( $p$-value=0.2985); however, a significant temperature effect was found in the bio-oil produced from W/E-HTL ( $p$-value=0.0001). The carbonyl concentration of bio-oils produced from W-HTL and W/E-HTL followed the same pattern under the temperature treatment: first increased (p-value $=0.1326$ and 0.0002 for W-HTL and W/E-HTL, respectively) as the HTL temperature was increased from $250{ }^{\circ} \mathrm{C}$ to $300{ }^{\circ} \mathrm{C}$, and then decreased ( $p$-value $=0.0022$ and 0.0099 for W-HTL and W/E-HTL, respectively) with increasing temperature from 300 to $350^{\circ} \mathrm{C}$. A slight increase in the carbonyl concentration (pvalue $=0.6216$ and 0.5658 for W-HTL and W/E-HTL, respectively) was observed when the HTL temperature had reached to $390^{\circ} \mathrm{C}$. The first increase in the carbonyl concentration could indicate the enhanced degradation of hemicellulose at $300^{\circ} \mathrm{C}$ since the major degradation product of hemicellulose is xylose which is a 5-carbon sugar aldehyde (Changi et al. 2015). Moreover, cleavage of ester bonds in lignin could result in the increase of carbonyl concentration (Huang et al. 2014) as lignin degradation mostly occurs at temperature above $260{ }^{\circ} \mathrm{C}$ (Yuan et al. 2010). Decrease in the carbonyl concertation with increasingly severe HTL conditions is more 
likely due to the dehydration (Changi et al. 2015) and re-condensation of carbonyl groups with other degradation products.

\subsubsection{Effect of Ethanol on Carbonyl $(C=O)$ Groups}

ANOVA analysis showed that addition of ethanol had significant effect on carbonyl concentration at each HTL temperature ( $p$-value $=0.0030,0.0020,0.0031$ and 0.0055 for 250 , 300,350 and $390^{\circ} \mathrm{C}$, respectively). Compared to W-HTL, a lower carbonyl concentration was found in the W/E-HTL bio-oils at each temperature (Fig. 4). This is in agreement with research by Kosinkova et al. (2015) who pursued the HTL of bagasse using water/ethanol solvent. In that study, the GC/MS analysis of bio-oil showed that the amount of ketones in the bio-oil decreased as the amount of ethanol was increased in the water/ethanol mixture. Acetalisation studies of biooil also showed that highly reactive carbonyl groups could be converted into more stable acetal and hemiacetal groups by low molecular weight alcohols such as methanol and ethanol (Li et al. 2011, Ye et al. 2014). Eckert and Chandler (1998) investigated the tautomeric equilibrium of Schiff base 4-(methoxy)-1-(N-phenylforminidoyl)-2-naphthol in the supercritical ethane in the presence of ethanol as the co-solvent, and reported that ethanol could shift the keto-enol equilibrium toward the enol form with increasing density which suggests that ethanol played a role in the conversion of ketone carbonyls to hydroxyl groups. Similar transformation of ketones to enols was also observed by Nazari et al. (2015) where birchwood sawdust was liquefied in the presence of $\mathrm{KOH}$ in a HTL process. Taking the reported observations into account, it is concluded that the lower carbonyl and higher hydroxyl concentration in bio-oil produced via W/E- HTL in this study could be attributed to (i) the stabilization of carbonyl groups to acetal groups by ethanol, and (ii) the shift of keto-enol tautomerism toward the enol form. 
Compounds containing carbonyl groups are considered undesirable for the utilization of bio-oil due to their high reactivity which leads to unstable, highly acidic bio-oil (Huang et al. 2014). The results of this study suggest that, especially at lower temperatures, carbonyl concentration could be significantly reduced by addition of ethanol to the HTL process. Such bio-oil with improved properties may open venues to increased utilization.

\section{Conclusion}

The effect of ethanol on hydroxyl and carbonyl groups in bio-oil was studied. The highest bio-oil yield was obtained from W/E-HTL at $300{ }^{\circ} \mathrm{C} .{ }^{31} \mathrm{P}-\mathrm{NMR}$ and ${ }^{19} \mathrm{~F}-\mathrm{NMR}$ showed that addition of ethanol had a significant effect on the total hydroxyl and carbonyl concentration of the bio-oils. It was found that addition of ethanol increased the total $\mathrm{OHN}$ and aliphatic type $\mathrm{OH}$; however, decreased the phenolic $\mathrm{OH}$ concentration. Moreover, lower carbonyl concentration was observed when ethanol was introduced to the HTL process. With optimization of the HTL reaction conditions the properties of bio-oils might be tailored towards their area of application.

\section{Acknowledgement}

The Auburn University Intramural Grants Program is recognized for startup funding for part of this project. Another part was supplied by NSF Auburn IGERT: Integrated Biorefining for Sustainable Production of Fuels and Chemicals (NSF Award \#: 1069004). This work was also supported by the Agriculture and Food Research Initiative (AFRI) CAP - "Southeast Partnership for Integrated Biomass Supply Systems” (Project \#: TEN02010-05061). Further, Regions Bank provided partial support and the Forest Products Development Center is acknowledged for supplementary funding of materials and supplies. The Center for Bioenergy and Bioproducts is acknowledged for use of their facilities. This work was also supported by the NSF under grant 
CREST \# HDR-1137681. Paula Davis and Rhonda Gibson are also thanked for their assistance with obtaining and maintaining the funds utilized for the research performed in this paper. 


\section{References}

1. Akhtar, J. and N. A. S. Amin. 2011. A review on process conditions for optimum bio-oil yield in hydrothermal liquefaction of biomass. Renewable and Sustainable Energy Reviews 15:1615-1624.

2. Ben, H. X. and A. J. Ragauskas. 2011. NMR Characterization of Pyrolysis Oils from Kraft Lignin. Energy \& Fuels 25:2322-2332.

3. Celikbag, Y., T. J. Robinson, B. K. Via, S. Adhikari, and M. L. Auad. 2015. Pyrolysis oil substituted epoxy resin: Improved ratio optimization and crosslinking efficiency. Journal of Applied Polymer Science 132. DOI: 10.1002/app.42239

4. Celikbag, Y., B. K. Via, S. Adhikari, and Y. Wu. 2014. Effect of liquefaction temperature on hydroxyl groups of bio-oil from loblolly pine (Pinus taeda). Bioresource Technology 169:808-811.

5. Changi, S. M., J. L. Faeth, N. Mo, and P. E. Savage. 2015. Hydrothermal Reactions of Biomolecules Relevant for Microalgae Liquefaction. Industrial \& Engineering Chemistry Research. 54:11733-11758.

6. Cheng, S., I. D'cruz, M. Wang, M. Leitch, and C. Xu. 2010. Highly Efficient Liquefaction of Woody Biomass in Hot-Compressed Alcohol-Water Co-solvents. Energy \& Fuels 24:4659-4667.

7. Choi, G.-G., S.-J. Oh, S.-J. Lee, and J.-S. Kim. 2015. Production of bio-based phenolic resin and activated carbon from bio-oil and biochar derived from fast pyrolysis of palm kernel shells. Bioresource Technology 178:99-107.

8. Czernik, S., D. K. Johnson, and S. Black. 1994. Stability of Wood Fast Pyrolysis Oil. Biomass \& Bioenergy 7:187-192.

9. Eckert, C. A. and K. Chandler. 1998. Tuning fluid solvents for chemical reactions. The Journal of Supercritical Fluids 13:187-195.

10. Elliott, D. C., P. Biller, A. B. Ross, A. J. Schmidt, and S. B. Jones. 2015. Hydrothermal liquefaction of biomass: Developments from batch to continuous process. Bioresource Technology 178:147-156.

11. Hu, S. J. and Y. B. Li. 2014. Two-step sequential liquefaction of lignocellulosic biomass by crude glycerol for the production of polyols and polyurethane foams. Bioresource Technology 161:410-415.

12. Huang, F., S. Pan, Y. Pu, H. Ben, and A. J. Ragauskas. 2014. 19F NMR spectroscopy for the quantitative analysis of carbonyl groups in bio-oils. Rsc Advances 4:17743-17747. 
13. Kosinkova, J., J. A. Ramirez, J. Nguyen, Z. Ristovski, R. Brown, C. S. K. Lin, and T. J. Rainey. 2015. Hydrothermal liquefaction of bagasse using ethanol and black liquor as solvents. Biofuels, Bioproducts and Biorefining. DOI: 10.1002/bbb.1578

14. Krammer, P. and H. Vogel. 2000. Hydrolysis of esters in subcritical and supercritical water. The Journal of Supercritical Fluids 16:189-206.

15. Kruse, A. and E. Dinjus. 2007. Hot compressed water as reaction medium and reactant: Properties and synthesis reactions. The Journal of Supercritical Fluids 39:362-380.

16. Kunaver, M., S. Medved, N. Cuk, E. Jasiukaityte, I. Poljansek, and T. Strnad. 2010. Application of liquefied wood as a new particle board adhesive system. Bioresource Technology. 101:1361-1368.

17. Kuo, P.-Y., M. Sain, and N. Yan. 2014. Synthesis and characterization of an extractivebased bio-epoxy resin from beetle infested Pinus contorta bark. Green Chemistry 16:3483-3493.

18. Li, X., R. Gunawan, C. Lievens, Y. Wang, D. Mourant, S. Wang, H. Wu, M. GarciaPerez, and C.-Z. Li. 2011. Simultaneous catalytic esterification of carboxylic acids and acetalisation of aldehydes in a fast pyrolysis bio-oil from mallee biomass. Fuel 90:25302537.

19. Liu, Y., X.-z. Yuan, H.-j. Huang, X.-1. Wang, H. Wang, and G.-m. Zeng. 2013. Thermochemical liquefaction of rice husk for bio-oil production in mixed solvent (ethanol-water). Fuel Processing Technology 112:93-99.

20. Nazari, L., Z. Yuan, S. Souzanchi, M. B. Ray, and C. Xu. 2015. Hydrothermal liquefaction of woody biomass in hot-compressed water: Catalyst screening and comprehensive characterization of bio-crude oils. Fuel 162:74-83.

21. Ouyang, X., X. Huang, Y. Zhu, and X. Qiu. 2015. Ethanol-Enhanced Liquefaction of Lignin with Formic Acid as an in Situ Hydrogen Donor. Energy \& Fuels 29:5835-5840.

22. Perlack, R. D., L. L. Wright, A. F. Turhollow, R. L. Graham, B. J. Stokes, and D. C. Erbach. 2005. Biomass as feedstock for a bioenergy and bioproducts industry: the technical feasibility of a billion-ton annual supply. DTIC Document.

23. Thangalazhy-Gopakumar, S., S. Adhikari, H. Ravindran, R. B. Gupta, O. Fasina, M. Tu, and S. D. Fernando. 2010. Physiochemical properties of bio-oil produced at various temperatures from pine wood using an auger reactor. Bioresource Technology 101:83898395.

24. Toor, S. S., L. Rosendahl, and A. Rudolf. 2011. Hydrothermal liquefaction of biomass: A review of subcritical water technologies. Energy 36:2328-2342. 
25. Valdez, P. J. and P. E. Savage. 2013. A reaction network for the hydrothermal liquefaction of Nannochloropsis sp. Algal Research 2:416-425.

26. Vasilakos, N. P. and D. M. Austgen. 1985. Hydrogen-donor solvents in biomass liquefaction. Industrial \& Engineering Chemistry Process Design and Development 24:304-311.

27. Via, B. K., S. Adhikari, and S. Taylor. 2013. Modeling for proximate analysis and heating value of torrefied biomass with vibration spectroscopy. Bioresource Technology 133:1-8.

28. Wei, N., B. K. Via, Y. F. Wang, T. McDonald, and M. L. Auad. 2014. Liquefaction and substitution of switchgrass (Panicum virgatum) based bio-oil into epoxy resins. Industrial Crops and Products 57:116-123.

29. Yan, X., B. Wang, and J. Zhang. 2015. Liquefaction of cotton seed in sub-critical water/ethanol with modified medical stone for bio-oil. Bioresource Technology 197:120127.

30. Ye, J., C. Liu, Y. Fu, S. Peng, and J. Chang. 2014. Upgrading Bio-oil: Simultaneous Catalytic Esterification of Acetic Acid and Alkylation of Acetaldehyde. Energy \& Fuels 28:4267-4272.

31. Ye, Y., J. Fan, and J. Chang. 2012. Effect of reaction conditions on hydrothermal degradation of cornstalk lignin. Journal of Analytical and Applied Pyrolysis 94:190-195.

32. Yu, F., Y. H. Liu, X. J. Pan, X. Y. Lin, C. M. Liu, P. Chen, and R. Ruan. 2006. Liquefaction of corn stover and preparation of polyester from the liquefied polyol. Applied Biochemistry and Biotechnology 130:574-585.

33. Yuan, X. Z., H. Li, G. M. Zeng, J. Y. Tong, and W. Xie. 2007. Sub- and supercritical liquefaction of rice straw in the presence of ethanol-water and 2-propanol-water mixture. Energy 32:2081-2088.

34. Yuan, Z., S. Cheng, M. Leitch, and C. Xu. 2010. Hydrolytic degradation of alkaline lignin in hot-compressed water and ethanol. Bioresource Technology 101:9308-9313.

35. Zou, X. W., T. F. Qin, Y. Wang, L. H. Huang, Y. M. Han, and Y. Li. 2012. Synthesis and properties of polyurethane foams prepared from heavy oil modified by polyols with 4,4 'methylene-diphenylene isocyanate (MDI). Bioresource Technology 114:654-657. 


\section{Figure Captions}

Fig. 1 Product separation procedure of HTL of loblolly pine.

Fig. 2 Product yields at different HTL conditions ( $\mathrm{W}=$ water, $\mathrm{W} / \mathrm{E}=$ =water/ethanol solvent).

Fig. $3 \mathrm{OHN}$ of bio-oils produced by HTL of loblolly pine at different conditions derivatized with TMDP.

Fig. 4 Carbonyl group concentration of bio-oils produced by HTL of loblolly pine at different conditions derivatized with 4-(tri-fluoromethyl)phenylhydrazine (W=water, W/E=water/ethanol solvent). 
Table 1. The initial and final pressure in the reactor at each temperature in HTL process.

\begin{tabular}{|c|c|c|c|c|}
\hline \multirow[b]{2}{*}{$\begin{array}{c}\text { Temperature } \\
\left({ }^{\circ} \mathrm{C}\right)\end{array}$} & \multicolumn{2}{|c|}{ Initial $\mathrm{N}_{2}$ Pressure $(\mathrm{MPa})$} & \multicolumn{2}{|c|}{ Final Pressure (MPa) } \\
\hline & $\mathrm{H}_{2} \mathrm{O}$ & $\mathrm{H}_{2} \mathrm{O} / \mathrm{EtOH}$ & $\mathrm{H}_{2} \mathrm{O}$ & $\mathrm{H}_{2} \mathrm{O} / \mathrm{EtOH}$ \\
\hline 250 & 2 & 2 & 7 & 9 \\
\hline 300 & 2 & 2 & 11 & 14 \\
\hline 350 & 2 & 2 & 19 & 22 \\
\hline 390 & 0 & 0 & 24 & 24 \\
\hline
\end{tabular}




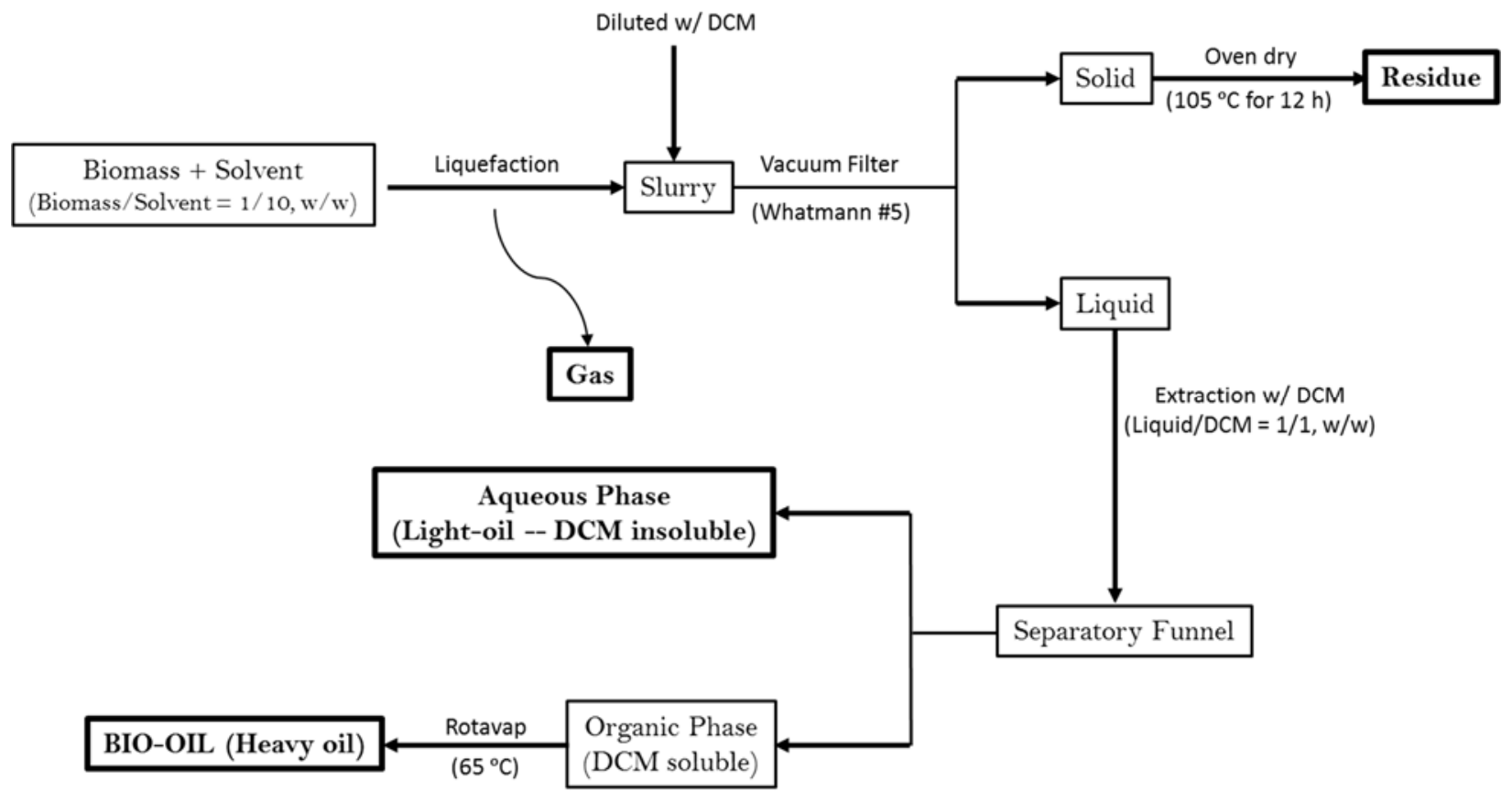

Fig. 1 Product separation procedure of HTL of loblolly pine. 


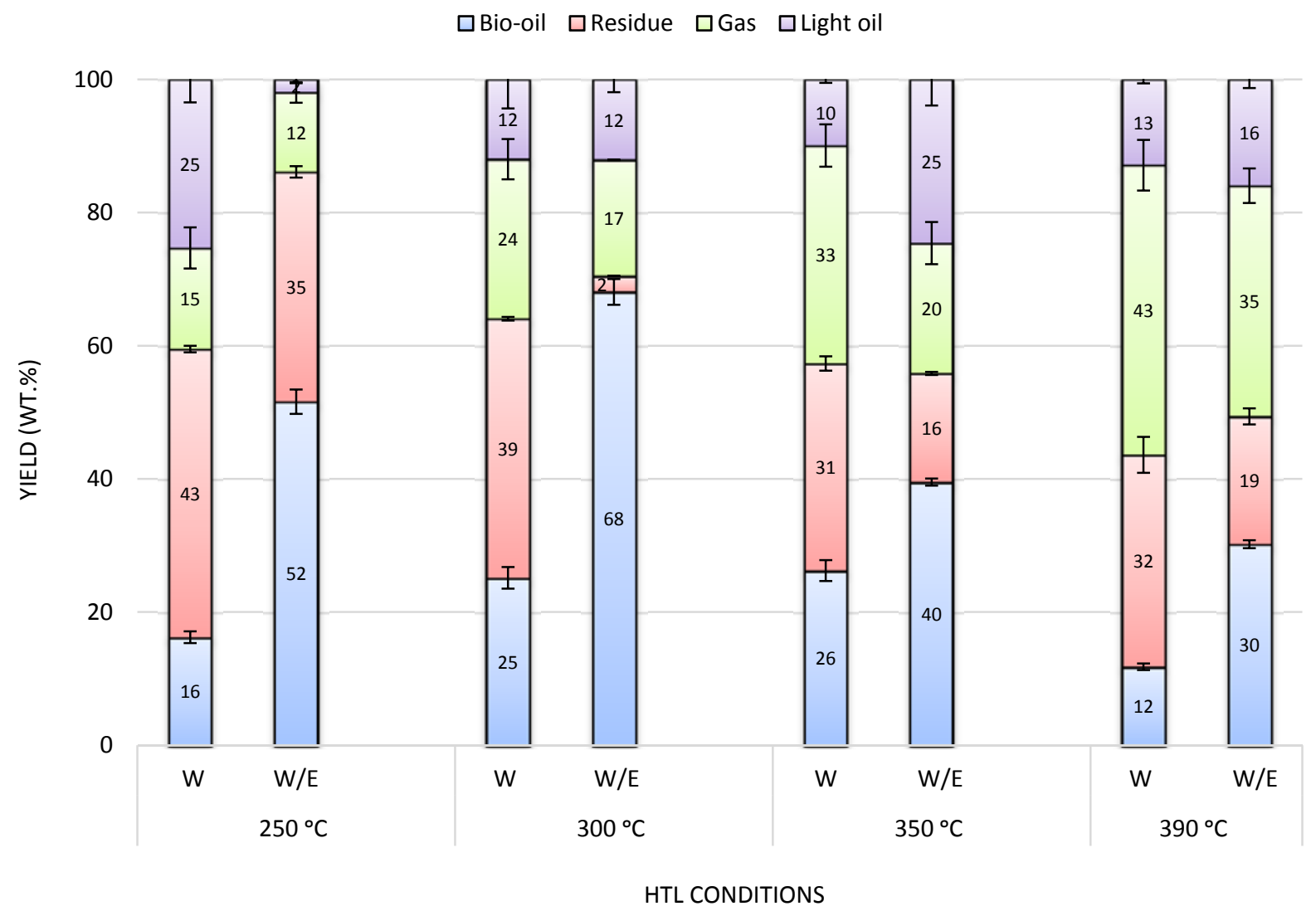

Fig. 2 Product yields at different HTL conditions ( $\mathrm{W}=$ water, $\mathrm{W} / \mathrm{E}=$ water/ethanol solvent). 


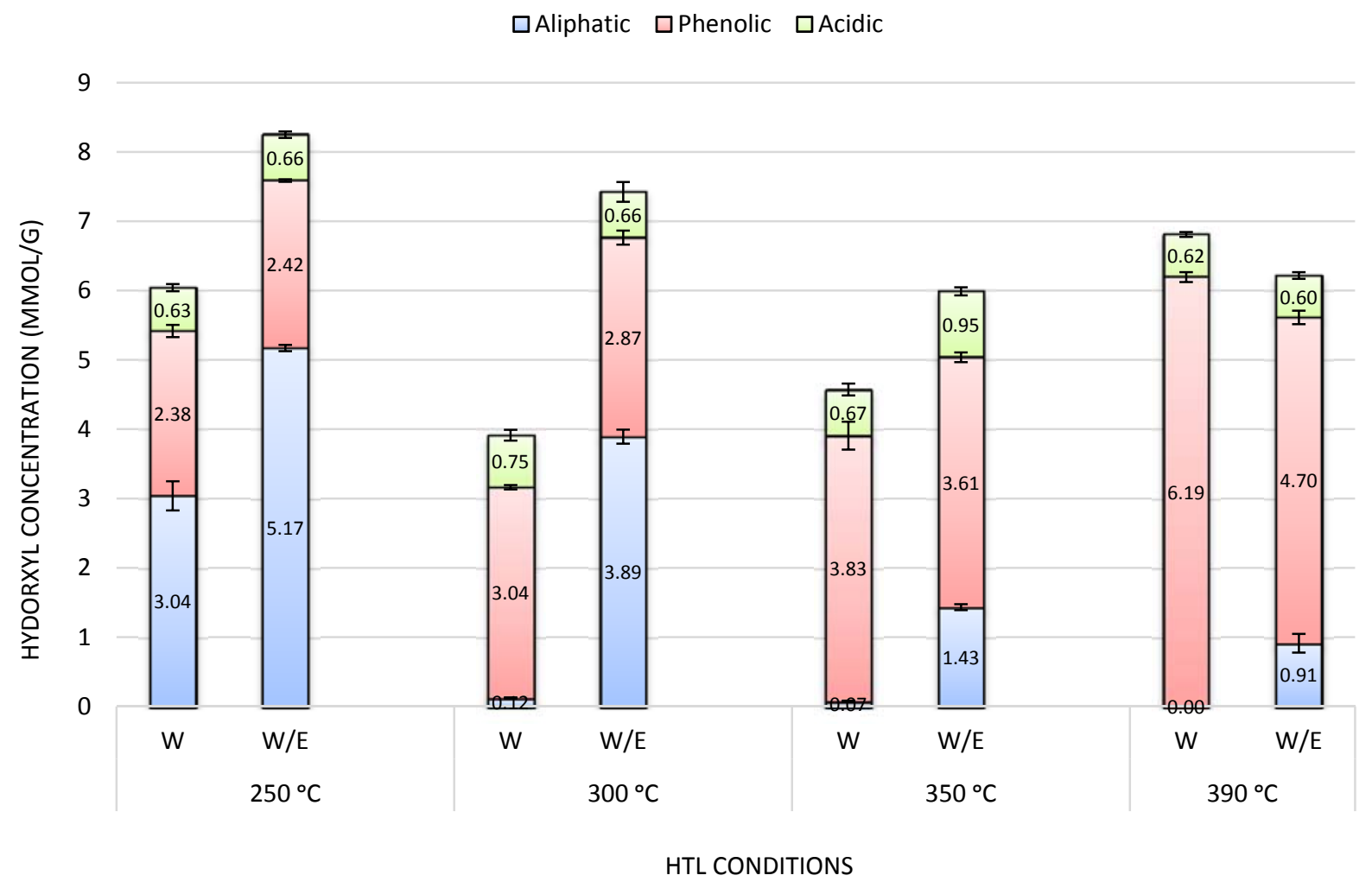

Fig. $3 \mathrm{OHN}$ of bio-oils produced by HTL of loblolly pine at different conditions derivatized with TMDP. 


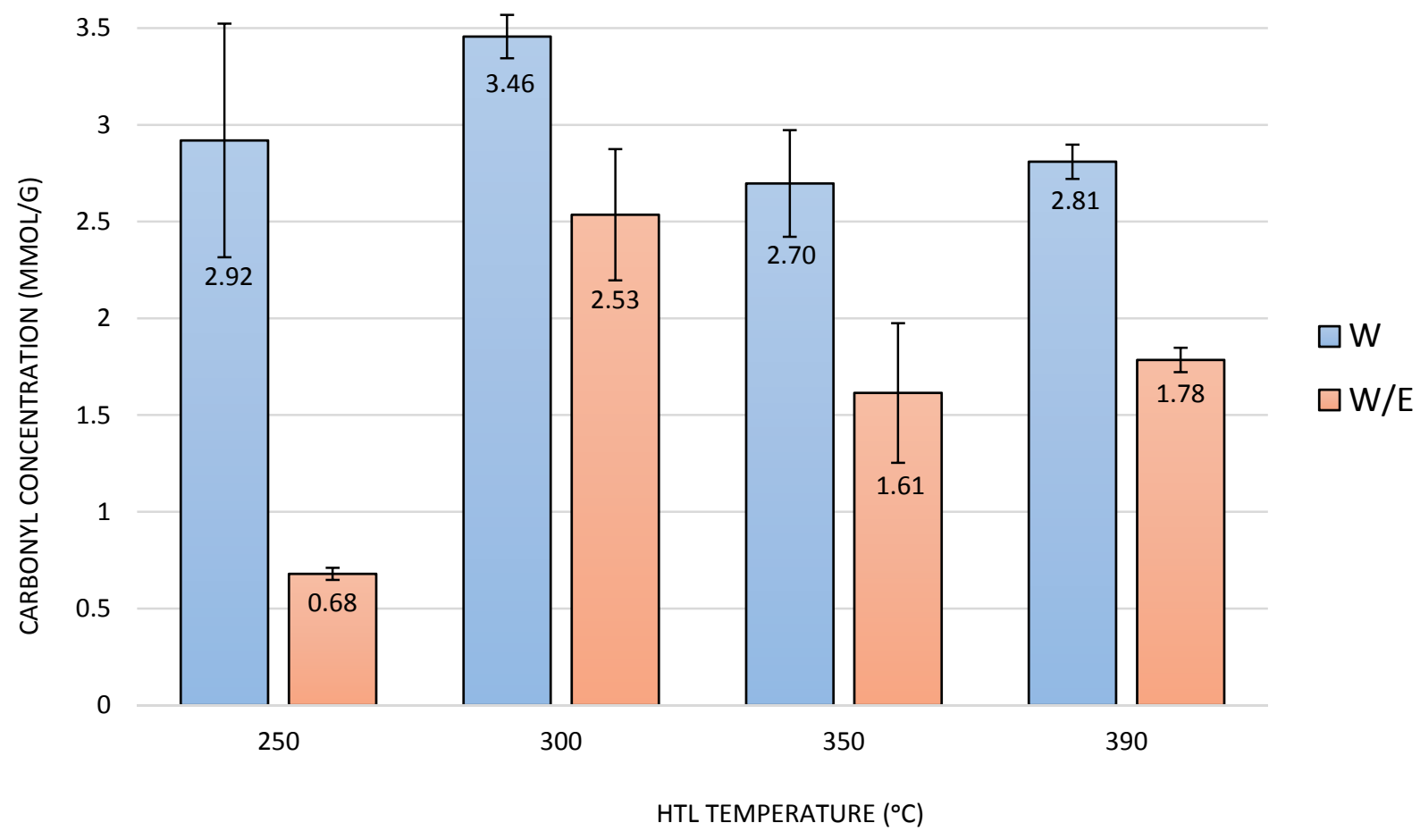

Fig. 4 Carbonyl group concentration of bio-oils produced by HTL of loblolly pine at different conditions derivatized with 4-(tri-fluoromethyl)phenylhydrazine (W=water, W/E=water/ethanol solvent). 


\section{Highlights}

- For the first time, ${ }^{31} \mathrm{P}-\mathrm{NMR}$ and ${ }^{19} \mathrm{~F}-\mathrm{NMR}$ were employed to understand the effect of ethanol on the formation bio-oil.

- Ethanol significantly increased the bio-oil and decreased the residue yield.

- Addition of ethanol increased the hydroxyl concentration while decreased the carbonyl concentration.

- More aliphatic and less phenolic type $\mathrm{OH}$ was obtained when ethanol was used as a cosolvent. 